\title{
Turbulent boundary layers at very large Reynolds numbers
}

\author{
G. I. Barenblatt \\ Professor-in Residence, Department of Mathematics \\ University of California at Berkeley, \\ and Lawrence Berkeley National Laboratory; \\ Adviser, Institute of Oceanology, \\ Russian Academy of Sciences
}

June 10, 2003

1. Turbulence is the state of vortex fluid motions where the properties of the flow field (velocity, pressure, etc.) vary in time and in space randomly.

Turbulent flows surround us, in the atmosphere, the oceans, in engineering and natural systems. First recognized, examined and even baptized by Leonardo da Vinci, turbulence has been studied more than a century by scientists and engineers, including the giants, Andrey Nikolaevich Kolmogorov, W. Heisenberg, G. I. Taylor, L. Prandtl, Th. von Kármán. Every advance in a wide collection of subjects, from chaos and fractals to field theory, and every increase in speed and parallelization of computers was heralded as ushering in the solution of the "turbulence problem". However, the turbulence, a phenomenon familiar now to everybody remains the greatest challenge of applied mathematics and classical physics. Turbulence at very large Reynolds numbers (often called developed turbulence) was widely considered to be one of the happier provinces of the turbulence realm, as it was widely thought that two of its basic results are well-established, and will enter, basically untouched, into a future pure self-contained theory of turbulence. These results are: the von Kármán-Prandtl universal logarithmic law for wall-bounded turbulent shear flows [1,2], and the Kolmogorov-Obukhov scaling laws for the local structure of developed turbulent flows, famous K-41 [3,4].

The beginning of the fundamental research of turbulent flows at very large Reynolds numbers can be dated sharply: it was the lecture of Th. von Kármán "Mechanical similitude and turbulence" at the Third International Congress for Applied Mechanics at Stockholm, in the end of August 1930 [1].

Von Kármán began his lecture with the following general statement: 
"Our experimental knowledge of the internal structure of turbulent flows is insufficient for delivering a reliable foundation for a rational theoretical calculation of the velocity distribution and drag in the so-called hydraulic flow state. Numerous semi-empirical formulae, for instance, the attempt to introduce turbulent drag coefficients, are unable to satisfy neither the theoretician, nor the practitioner... I will restrict myself rather to clarifying what can be achieved on the basis of pure fluid dynamics if definite hypotheses are introduced concerning definite basic questions."

More than fifty years later, surveying in the end of his life the beginning of his work in turbulence, Andrey Nikolaevich said:

"It became clear for me that it is unrealistic to have a hope for the creation of a pure self-contained theory [of the turbulent flows of fluids and gases]... Due to absence of such a theory we have to rely upon the hypotheses, obtained by processing experimental data... I did not perform an experimental work by myself, but I spent a lot of energy for calculations and graphical processing of the data by other researchers."

Thus, both von Kármán and Andrey Nikolaevich had thought that it is unrealistic to expect in a real time the creation of a pure general theory of turbulence closed in itself. Both of them considered as a realistic and sound path to rely upon the hypotheses, suggested by experiments, and therefore valid for special classes of flows only.

What we can say now, after many decades? Very little, if anything, has changed as far as pure general theory is concerned. It is very discouraging that in spite of hard work by an army of scientists during more than a century almost nothing became known about turbulence from first principles: the Navier-Stokes equations and the continuity equation.

However, something has changed, and changed drastically. Now we have in public possession a larger amount of much more reliable experimental data. We are able, processing these data, to refine, and to modify, if necessary, the basic hypotheses and special theories based on them.

It is difficult nowadays to discuss the local structure of turbulence at very high Reynolds numbers. The reason is simple: a clean laboratory experimental database is not yet sufficient to come to definite conclusions. The geophysical (atmospheric and oceanic) data are not sufficiently clean. I personally believe that K-41, the Kolmogorov-Obukhov scaling laws in their classical formulation describe a large class of fluid flows in nature. Andrey Nikolaevich Kolmogorov, A. M. Obukhov, a little later independently W. Heisenberg and L. Onsagerwhat great names! - have selected this topic, very unusual for that time and established these laws. Such beauty cannot be useless!

I will discuss in this lecture wall-bounded turbulent shear flows (von Kármán called them hydraulic state) at very large Reynolds numbers. The work which I will speak about was 
performed basically in 1991-2002 by A. J. Chorin, V. M. Prostokishin and myself. The reader can be referred to the papers [22-25] which contain also further earlier references.

We claim that the experimental data, obtained in the last decades and available in public possession contradict the basic hypothesis proposed by Th. von Kármán. Therefore, the universal logarithmic law is not quite correct and should be abandoned. The subsequent theories based on the universal logarithmic law should be reconsidered. We propose instead a Reynolds-number-dependent scaling law for the velocity distribution in wall-bounded shear flow and drag law corresponding to it.

2. We turn now to the problem of our direct interest: statistically steady wall-bounded turbulent shear flows. Among such flows there are many flows of practical importance, such as flows in pipes, channels and boundary layers.

Flows in cylindrical pipes (Figure 1) give an instructive example. We have the same clear goal and well determined problems as ones formulated by von Kármán in his lecture: to obtain the mathematical expression for the velocity distribution in the intermediate region of the flow, and for the drag coefficient. "Intermediate" for the pipe flows means the region between the "viscous sublayer" adjacent to the wall in which the velocity gradients are so high that the viscous stress is comparable with the stress created by the turbulent vortices, and a close vicinity of the pipe axis. For other wall-bounded flows the outer boundary of the intermediate region will be defined in due place.

The hypothesis proposed by von Kármán was presented by him in a following straightforward form:

"On the basis of these experimentally well established facts we make an assumption that outside a close vicinity of the wall the velocity distribution of the mean flow is viscosity independent."

It was clear that the velocity gradient $\partial_{y} u$ not the velocity $u$ itself was assumed to be viscosity independent, apparently it was explicitly formulated by Landau [6].

Consider now the derivation of the velocity distribution in the intermediate region based on this hypothesis. The mean velocity gradient $\partial_{y} u$ depends on the following arguments: the transverse coordinate $y$ (the distance from the wall), the shear stress at the wall $\tau$, the pipe diameter $d$, and the fluid properties: its kinematic viscosity $\nu$ and density $\rho$. The velocity gradient $\partial_{y} u$ is considered rather than the velocity $u$ by itself because the values of $u$ at any distance from the wall depend on the flow in the vicinity of the wall (viscous sublayer) where the assumptions asymptotic in their nature which will be used further are invalid. Thus

$$
\partial_{y} u=f(y, \tau, d, \nu, \rho) .
$$

Following von Kármán and Prandtl we introduce the viscous length scale

$$
\delta=\nu / u_{*}, \text { where } u_{*}=(\tau / \rho)^{1 / 2} .
$$


The quantity $u_{*}$ is called dynamic, or 'friction' velocity. The dimensional analysis gives

$$
\partial_{y} \phi=\frac{1}{y} \Phi\left(\frac{y}{\delta}, \frac{d}{\delta}\right), \phi=\frac{u}{u_{*}} .
$$

Also, the dimensional analysis shows that $d / \delta$ is a function of the traditional Reynolds number $\operatorname{Re}=\bar{u} d / \nu$, where $\bar{u}$ is the average velocity - the total flux per unit time divided by the cross-section area of the pipe. Thus, the relation (3) can be written in the form

$$
\partial_{y} \phi=\frac{1}{y} \Phi(\eta, \mathrm{Re}), \eta=\frac{y}{\delta}=\frac{u_{*} y}{\nu} .
$$

The laminar sublayer occupies several tens of viscous lengths, therefore for very large Reynolds numbers in the intermediate region the argument $y / \delta$ is large. The basic von Kármán hypothesis is that the viscosity does not affect the velocity (in fact, the velocity gradient) distribution in the intermediate region. Meanwhile the viscosity enters both arguments of the function $\Phi$ in (4). Thus, according to the von Kármán hypothesis the viscous length scale $\delta$ should disappear from the resulting relation and the function $\Phi$ must be replaced by a constant: $\Phi=1 / \kappa$. The constant $\kappa$ was later named the 'Kármán constant'. Substituting $\Phi=1 / \kappa$ to (4) gives

$$
\partial_{y} \phi=\frac{1}{\kappa y}, \phi=\frac{u}{u_{*}} .
$$

The subsequent integration gives the von Kármán-Prandtl 'universal' (Reynolds-numberindependent) logarithmic law for the velocity distribution:

$$
\phi=\frac{u}{u_{*}}=\left[\frac{1}{\kappa} \ln \eta+C\right], \eta=\frac{u_{*} y}{\nu} .
$$

Here the constant $C$,- and this is also a seemingly logically consistent, but in fact additional substantial assumption,- - is finite and Reynolds number independent. By the very logic of the derivation the constants $\kappa$ and $C$ should be identical for all high quality experiments.

However, for more than six decades the experimental information has accumulated suggesting doubts in the universal logarithmic law (6). We emphasize that the only place in the derivation of (6) which can provoke doubts is the basic von Kármán hypothesis. In fact, even if a very liberal approach to the constants $\kappa$ and $C$ is allowed (for $\kappa$ - the values from 0.38 to 0.44 , i.e. $1 / \kappa$ from 2.25 to 2.65 were proposed, for $C$ - from 4.1 to 6.3 ) the region of validity of the law (6) happened to be very short. It is enough to mention here the data presented in the book [7] (p. 273), and, specially more recent data for pipe flows obtained by M. Zagarola at Princeton [8] (see Figure 2). The splitting of curves corresponding to 
different values of the Reynolds number is clearly seen, and the deviation from the straight line representing in the plane $\ln \eta, \phi$ the universal logarithmic law is a systematic one.

3. Before proceeding further, a general remark concerning the scaling laws will be useful. Consider a physical relationship (i.e. one valid for all observers having different magnitude of units of measurement):

$$
a=f\left(a_{1} \ldots a_{k}, b\right)
$$

where the arguments $a_{1}, \ldots, a_{k}$ have independent dimensions, whereas the dimensions of $a$ and $b$ can be expressed via the dimensions of $a_{1}, \ldots, a_{k}$ :

$$
[a]=\left[a_{1}\right]^{p} \ldots\left[a_{k}\right]^{r},[b]=\left[a_{1}\right]^{p_{1}} \ldots\left[a_{k}\right]^{r_{1}} .
$$

The relation (7) can be represented in a universal, dimensionless form valid for all observers having different by magnitude units of measurement:

$$
\Pi=\Phi\left(\Pi_{1}\right), \text { where } \Pi=\frac{a}{a_{1}^{p} \ldots a_{k}^{r}}, \Pi_{1}=\frac{b}{a_{1}^{p_{1}} \ldots a_{k}^{r_{1}}} .
$$

Now, if the value of the parameter $\Pi_{1}$ is, for definiteness sake, large, and there exists a finite non-zero limit of $\Phi\left(\Pi_{1}\right)$ at $\Pi_{1} \rightarrow \infty$, then the law (7) at sufficiently large $\Pi_{1}$ can be represented as a scaling law:

$$
a=C a_{1}^{p} \ldots a_{k}^{r}
$$

where all exponents can be obtained by the dimensional analysis, and the quantity $b$ ceases to influence the phenomenon. We refer to such cases as complete similarity (cf. an established term "Reynolds number similarity"). However, what can be said if such finite non-zero limit does not exist? Generally speaking - nothing. However, there exists an important case, which is wider than complete similarity, although also special. Remember that in fact the researcher is interested not in the limit, but in an asymptotics at large, although finite $\Pi_{1}$. So, if the finite non-zero limit of $\Phi\left(\Pi_{1}\right)$ at $\Pi_{1} \rightarrow \infty$ does not exist, but the asymptotics of $\Phi\left(\Pi_{1}\right)$ at $\Pi_{1} \rightarrow \infty$ is a power one: $\Phi=C \Pi_{1}^{\alpha}$ we obtain a scaling law of seemingly the same form as (10)

$$
a=C a_{1}^{p-\alpha p_{1}} \ldots a_{k}^{r-\alpha r_{1}} b^{\alpha} .
$$

The difference between (10) and (11) is that in (11) the exponents cannot be obtained by the dimensional analysis, because the number $\alpha$ is a priori unknown, and the quantity $b$ remains in (11), although in a monomial combination with other arguments. We refer to such cases as incomplete similarity. (In fact, here is presented a very special case of the 
incomplete similarity which will be needed further, for a general discussion the reader can be referred to [9].)

4. A strong argument for assuming incomplete similarity for pipe flows is available in the book by Schlichting [10]. Along with the full respect to the universal logarithmic law in this book is presented a series of graphs as the "verification of the assumption" of the Reynolds-number-dependent power law for the velocity distribution in pipes (see Figure 3). These graphs show that the data of the experiments of J. Nikuradze [11] which were generally considered (also by Prandtl himself) as the confirmation of the universal logarithmic law (6) can be represented with astonishing accuracy practically over the whole pipe cross-section by a power law with Reynolds-number-dependent exponent.

Therefore a natural step was to assume that in the case of wall-bounded turbulent shear flows there is no complete similarity corresponding to the von Kármán hypothesis, and to suggest the next by complexity hypothesis:

First hypothesis: there is an incomplete similarity of the mean velocity gradient in the parameter $y / \delta=u_{*} y / \nu=\eta$, and no kind of similarity in the Reynolds number.

According to this hypothesis, the influence of the viscosity remains at arbitrary large Reynolds numbers in the whole body of the flow, not only in the viscous sublayer. After the works of S. Kline, W. Reynolds and their group at Stanford (see [12] and many subsequent papers of these authors) the physical mechanism of the viscosity penetration into the flow core is clear: It is the penetration of vortices from the viscous sublayer to the main body of the flow. Truly remarkable is that this mechanism was anticipated by Prandtl much earlier. At the discussion immediately following the presentation of the von Kármán's lecture [1] he said that the disagreement with von Kármán formulae can be "attributed to the action of the viscosity also in the inner part of the flow, i.e. to the viscosity influenced streaks of which laminar layer at the wall consists, and which in this case enter far into the inner part of the flow". As far as it is known to the present author, this comment was never repeated neither by Prandtl himself, nor by somebody from Prandtl's inner circle!

Incomplete similarity means that the viscosity enters only in combination with other parameters controlling the turbulence. According to the first hypothesis for very large Re the function $\Phi$ entering the basic relation (4) at large $\eta=y / \delta=u_{*} y / \nu$ should be a power function of its argument $y / \delta=\eta$, while no special suggestion of any kind of similarity in the Reynolds number Re is assumed, so that

$$
\Phi\left(\frac{y}{\delta}, \operatorname{Re}\right)=A(\operatorname{Re})\left(\frac{y}{\delta}\right)^{\alpha(\operatorname{Re})}
$$

where $A(\mathrm{Re})$ and $\alpha(\mathrm{Re})$ are certain dimensionless functions which should be determined, $\delta=\nu / u_{*}$. 
From (12) and (4) we obtain

$$
\partial_{y} \phi=\frac{1}{y} A(\operatorname{Re})\left(\frac{u_{*} y}{\delta}\right)^{\alpha(\operatorname{Re})},
$$

where, we remind, $\phi=u / u_{*}$.

Our second hypothesis will be the vanishing-viscosity principle:

Second hypothesis: The gradient of average velocity tends to a well defined limit as the viscosity vanishes.

According to this principle it is possible to expand $A(\mathrm{Re})$ and $\alpha(\mathrm{Re})$ into a series in a small parameter $\varepsilon(\mathrm{Re})$ vanishing at $\mathrm{Re} \rightarrow \infty$, and to retain first two terms: $A=A_{0}+A_{1} \varepsilon$, $\alpha=\alpha_{0}+\alpha_{1} \varepsilon$, where $A_{0}, A_{1}, \alpha_{0}$ and $\alpha_{1}$ should be by the logic of derivation universal constants. We obtain from (13):

$$
\partial_{y} \phi=\frac{1}{y}\left(A_{0}+A_{1} \varepsilon\right)\left(\frac{y}{\delta}\right)^{\alpha_{0}+\alpha_{1} \varepsilon} .
$$

When the viscosity (and consequently the length scale $\delta$ ) goes to zero, a well-defined limit of $\partial_{y} \phi$ can exist for $\alpha_{0}=0$ only. Therefore, according to the second hypothesis $\alpha_{0}$ is equal to zero. Furthermore, (14) can be represented in the form:

$$
\partial_{y} \phi=\frac{1}{y}\left(A_{0}+A_{1} \varepsilon\right) \exp \left(\alpha_{1} \varepsilon \ln \frac{y}{\delta}\right) .
$$

The small parameter $\varepsilon$ is a function of Re, vanishing at $\mathrm{Re}=\infty$. Now - a very important point. The relation (15) shows that if $\varepsilon$ tends to zero at $\operatorname{Re} \rightarrow \infty$ faster than $1 / \ln \operatorname{Re}$, the argument of the exponent in (15) tends to zero, and we return to the case of complete similarity. As we have seen, the experiments show that this is not the case. If $\varepsilon$ tends to zero slower than 1/ln Re, the well-defined limit of the velocity gradient at the viscosity going to zero does not exist, and we obtain a contradiction to our second hypothesis-vanishing viscosity principle. Therefore the only choice compatible with the basic hypotheses is $\varepsilon=$ $1 / \ln$ Re, and we obtain by integration of (15) the scaling law for the velocity distribution in the intermediate region:

$$
\phi=\frac{u}{u_{*}}=\left(C_{0} \ln \operatorname{Re}+C_{1}\right)\left(\frac{y}{\delta}\right)^{\alpha_{1} / \ln \operatorname{Re}} .
$$

Note that here an additional condition $\phi(0)=0$ was used. This condition is an independent assumption, confirmed by experiments (see the Figure 3), which does not follow from the non-slip condition $u(0)=0$, because the boundary $y=0$ is outside the range of applicability of the asymptotic relation (15). 
The constants $C_{0}, C_{1}$ and $\alpha_{1}$ were obtained by comparison of the law (16) with the data of classic experiments by Nikuradze [11] performed under direct guidance of Prandtl. The following values were obtained

$$
C_{0}=\frac{1}{\sqrt{3}}, C_{1}=\frac{5}{2}, \alpha_{1}=\frac{3}{2} .
$$

Therefore the ultimate scaling law proposed for the velocity distribution in the basic, intermediate region of the pipe (see Figure 1) takes the form

$$
\phi=\left(\frac{\sqrt{3}+5 \alpha}{2 \alpha}\right) \eta^{\alpha}, \alpha=\frac{3}{2 \ln \operatorname{Re}}, \phi=\frac{u}{u_{*}}, \eta=\frac{u_{*} y}{\nu} .
$$

Note a clear-cut qualitative difference between the cases of complete similarity (equation (6)), and incomplete similarity (equation (18)). In the first case in the traditional $\ln \eta, \phi$ plane the experimental points should cluster along the single straight line of the universal logarithmic law. In the second case the experimental points should occupy an area in $\ln \eta, \phi$ plane, and to each value of Re corresponds a separate curve. The experimental data presented in the Figure 2 confirm that this is the case. Thus, the scaling law (18) shows that instead of universal Re-independent curve for the velocity distribution in the $\ln \eta, \phi$ plane dictated by the universal logarithmic law there is a family of curves in the $\ln \eta, \phi$ plane with Re as a parameter.

5. A transparent mathematical example proposed by A. J. Chorin clarifies what happens in the velocity field of wall-bounded turbulent shear flows. Consider a family of curves

$$
\phi=\left(\ln \frac{d}{\delta}\right)\left(\frac{y}{\delta}\right)^{1 / \ln (d / \delta)}-2 \ln \frac{d}{\delta} .
$$

Here $\phi$ is a dimensionless function, $d$ and $\delta$ are constant parameters having the dimension of length, $y \geq \delta$. We assume that $d$ is fixed, and $\delta$ is the parameter of the family.

The family (19) satisfies an ordinary differential equation

$$
\frac{d^{2} \phi}{d y^{2}}=\left(\frac{1}{\ln (d / \delta)}-1\right) \frac{1}{y} \frac{d \phi}{d y}
$$

and the boundary conditions at $y=\delta$

$$
\phi(\delta)=-\ln (d / \delta),\left.\frac{d \phi}{d y}\right|_{y=\delta}=\frac{1}{\delta} .
$$


Assume now that $d \gg \delta$, so that $1 / \ln (d / \delta)$ is a small parameter. For the curves of the family (19) a relation is valid:

$$
y \partial_{y} \phi=\left(\frac{y}{\delta}\right)^{1 / \ln (d / \delta)}=\exp \left[\frac{\ln (y / d)+\ln (d / \delta)}{\ln (d / \delta)}\right],
$$

so that at $d / \delta \rightarrow \infty$ and fixed $y / d$ the quantity $y \partial_{y} \phi$ tends to $e$. The family (19) has an envelope

$$
\phi=\ln (y / d),
$$

for which the quantity $y \partial_{y} \phi$ is also a constant, but a different one, equal to 1 . Let now neglect in the equation (20) the small parameter $1 / \ln (d / \delta)$ in comparison with 1 , then this equation is reduced to the form

$$
\frac{d^{2} \phi}{d y^{2}}=-\frac{1}{y} \frac{d \phi}{d y} .
$$

Solving this equation under boundary conditions (21) we obtain a single, $\delta$-independent "universal" curve, the envelope (23), and not the whole family (19). We can say, that neglecting small parameter in he equation (20) we "prevented" the penetration of the influence of the parameter $\delta$ into the basic region $y>\delta$, as it was done by the von Kármán hypothesis, and this changed the whole picture.

Let us look now on this matter from the viewpoint of the dimensional analysis. Dimensional analysis gives for the derivative $d \phi / d y$

$$
\frac{d \phi}{d y}=\frac{1}{y} \Phi\left(\frac{y}{\delta}, \frac{d}{\delta}\right)
$$

where $\Phi$ is a dimensionless function. In our case $\Phi=(y / \delta)^{1 / \ln d / \delta}$, so that at arbitrarily large $y / \delta$ the function $\Phi$ cannot be replaced by a constant. Therefore the influence of $\delta$ is preserved at arbitrarily large $y / \delta$ and cannot be neglected. However $\delta$ enters the resulting equation (19) in a specific form, in combination with $y$ and $d$ due to specific form of invariance of the problem as a whole.

6. Thus, I repeat, the scaling law (18) shows that instead of universal Re-independent single curve for the velocity distribution in the $\ln \eta, \phi$ plane there appears in this plane a family of curves having Reynolds number as a parameter. However, the family (18) has a special property of self-similarity, and therefore of universality. Indeed, if instead of $\phi$ we plot on the ordinate axis the quantity

$$
\psi=\frac{1}{\alpha} \ln \frac{2 \alpha \phi}{\sqrt{3}+5 \alpha}, \alpha=\frac{3}{2 \ln \operatorname{Re}},
$$


we obtain from (18) $\psi=\ln \eta$, i.e. the equation of the bisectrix of the first quadrant in the $\ln \eta, \psi$ plane. It is a crucial moment: whether the experiments will confirm it, yes or not? Processing of all Nikuradze's experimental data, available, I emphasize in the form of tables showed (see Figure 4) that indeed, the overwhelming majority of the experimental points for $\eta>30$ settled down along the bisectrix. The points corresponding to $\eta<30$ deviate from the bisectrix, and this is natural, because the scaling law describes the velocity distribution in the intermediate part of the cross-section, and the values $\eta>30$ correspond to the viscous sublayer. Note that there is a systematic deviation, not a scatter.

7. The scaling law (18) allows one to solve the second problem outlined by von Kármán for the flows in pipes - to find the drag. The drag is determined by the stress at the wall $\tau$, and the dimensionless drag coefficient is defined in a way: $\lambda=\tau /\left(\rho \bar{u}^{2} / 8\right)=8\left(u_{*}^{2} / \bar{u}^{2}\right)$, now common in the literature. We use for the determination of the average (over the crosssection) velocity $\bar{u}$ the scaling law (18), and so we neglect the deviation of the velocity distribution from the scaling law (18) both in the viscous sublayer and near the axis. We obtain the formula for the drag coefficient as a function of the Reynolds number:

$$
\lambda=\frac{8}{\Psi^{2 /(1+\alpha)}}, \Psi=\frac{e^{3 / 2}(\sqrt{3}+5 \alpha)}{2^{\alpha} \alpha(1+\alpha)(2+\alpha)}, \alpha=\frac{3}{2 \ln \operatorname{Re}} .
$$

Comparison of this law with an independent series of experiments where the friction was determined also shows an instructive agreement. The deviations are within the limits of a normal experimental scatter (see [22], [23] and the earlier references available in these papers).

We come to the conclusion that the scaling law (18) with universal, Reynolds number independent constants (17) and the drag law (27) describe accurately the turbulent flows in smooth pipes for large Reynolds numbers, and that the incomplete similarity of these flows can be considered as established. We emphasize that the values of the constants (17) should be considered as fixed, non-adjustable parameters.

8. The universal logarithmic law hardened into dogma, and became one of the pillars of the turbulence theory, and a mainstay of engineering science to a large extent because it was supported by an independent mathematical derivation based on seemingly unassailable principles. This derivation was proposed by A. Izakson [13], C. B. Millikan [14] and R. von Mises [15].

Our analysis showed that the Izakson-Millikan-von Mises derivation is not quite correct, and had to be modified taking into account the influence of the Reynolds number (see [22], [23]). In addition to splitting of the curves corresponding to different Reynolds numbers, a characteristic feature of the scaling law (18) is the availability of the straight line parts of these curves in $\ln \eta, \phi$ plane. Namely this property allows to modify the Izakson-Millikanvon Mises arguments. It is clearly supported by the experiments of Zagarola (see Figure 2). 
Also the family of power-laws (18) having Re as a parameter possesses an envelope (cf. Chorin's example presented in the Section 5). The relation for the envelope is obtained in an implicit form by elimination of Re from the equation (18), and an equation obtained from (18) by its differentiation over Re. And the envelope has an important feature: it is practically indistinguishable in the working range of $\ln \eta$ from the straight line

$$
\phi=\frac{u}{u_{*}}=\frac{\sqrt{3} e}{2} \ln \eta+5.1 .
$$

Bearing in mind that $2 / \sqrt{3} e \simeq 0.425$, the straight line $(28)$ can be identified with the traditional form of the universal logarithmic law. Therefore, if one plots the experimental points that correspond to various values of Re on a single graph in the $\ln \eta, \phi$ plane, what is natural for those who happen to believe the universal logarithmic law, that the envelope will be revealed. The visual impact of the envelope, when plotting the experimental data in the $\ln \eta, \phi$ plane, is magnified by the fact that the measurements at very small values of $\eta$, where the difference between the power laws and the envelope can also be noticeable are missing due to experimental difficulties.

We summarize: the confirmation of the universal logarithmic law by experiment for the pipe flows seems to be nothing but an illusion. Another fact in favour of this conclusion: an asymptotic analysis shows that at large Re the slopes of straight line parts of the curves in $\ln \eta, \phi$ plane make a discrepancy about $\sqrt{e}$ with the slope of the envelope (28), and this is also in agreement with experiment (see Figure 2).

9. By the very logic of its derivation, the scaling law (18) should be valid for any wallbounded shear flows, not only for the flows in pipes.

Here, however, the following question appears: What should be the definition of the Reynolds number for these flows which will allow us to apply to them the scaling law (18)? This question was immaterial as long as the researcher continues to believe in the universal logarithmic law: Indeed, if the law is Re-independent, the definition of Re does not matter. But the situation is completely different when the law is Re-dependent!

We will consider below the boundary layers, and we will show that the law (18) describes these flows too, if the Reynolds number is defined in an appropriate way.

Zero-pressure-gradient boundary layers were investigated by many experimentalists during last decades. The common choice of the Reynolds number for these flows is $\operatorname{Re}_{\theta}=U \theta / \nu$. Here $U$ is the free stream velocity, and $\theta$ is the momentum thickness, a quantity calculated by an integration of the velocity profile. This choice is rather arbitrary, and definitely the validity of the scaling law (18) with $\operatorname{Re}=\operatorname{Re}_{\theta}$ a priori cannot be expected. But what is the correct choice of the Reynolds number for the boundary layers?

To answer this question we have first of all to confirm that in the intermediate layer of the turbulent boundary layer adjacent to the viscous sublayer a certain scaling law is valid. To do that (see the details in [25]) all available experimental data which usually are presented 
in the traditional $\ln \eta, \phi$ plane were replotted in the bilogarithmic plane $\lg \eta, \lg \phi$. The result happened to be instructive: Without any exception for all investigated flows the straight lines were obtained for the region (I) adjacent to the viscous sublayer (see same examples in Figure 5). The straight line (I) corresponds to a scaling law

$$
\phi=A \eta^{\alpha}
$$

where the coefficients $A$ and $\alpha$ were obtained for each case by a statistical processing.

We take the Reynolds number in the form $\operatorname{Re}=U \Lambda / \nu$, where, we repeat, $U$ is the free stream velocity, and $\Lambda$ is a certain length scale. The question is whether such a unique length scale $\Lambda$ does exist, which plays for the intermediate region (I) of the boundary layer the same role as the pipe diameter for the pipe flow, i.e. such that the scaling law (18) is valid for the intermediate region (I)? To answer this question two values $\ln R_{1}$ and $\ln R_{2}$ were calculated by solving the equations suggested by the scaling law (18):

$$
\frac{1}{\sqrt{3}} \ln \operatorname{Re}_{1}+\frac{5}{2}=A, \frac{3}{2 \ln \operatorname{Re}_{2}}=\alpha,
$$

where, we repeat, $A$ and $\alpha$ were obtained by a statistical processing of the experimental data in the first intermediate self-similar region. The question was: whether the values $\ln \operatorname{Re}_{1}$ and $\ln \mathrm{Re}_{2}$, obtained by solving two different equations are close? Again: yes or not?

Analysis of all available experimental data [25] showed that indeed, the values of $\ln \mathrm{Re}_{1}$ and $\ln \mathrm{Re}_{2}$ are close, the difference between them as a rule does not exceed one-two percent. Therefore it is possible to introduce the mean Reynolds number $R e=\sqrt{R_{1} R_{2}}$, $\ln R e=$ $\left(\ln \mathrm{Re}_{1}+\ln \mathrm{Re}_{2}\right) / 2$ as an estimate for the effective Reynolds number of the boundary layer flow. After that we have to check the scaling law (18) in its universal form

$$
\psi=\frac{1}{\alpha} \ln \left(\frac{2 \alpha \phi}{\sqrt{3}+5 \alpha}\right)=\ln \eta
$$

using the experimental data for $\phi$ and $\eta$, and assuming $\alpha=3 / 2 \ln \operatorname{Re}, \ln \operatorname{Re}=\left(\ln \operatorname{Re}_{1}+\ln \operatorname{Re}_{2}\right) / 2$. According to (31) if our construction is correct, then in the coordinates $\ln \eta, \psi$ the experimental points should collapse onto the bisectrix of the first quadrant, as it was in the case of the flow in pipes. In Figure 6 the examples of such processing are presented which show that, indeed, this is the case. The results of similar processing of different data can be found in [25]. We conclude that scaling law (18) gives an accurate description of the mean velocity distribution over the self-similar region (I) adjacent to the viscous sublayer for a wide variety of zero-pressure-gradient boundary layer flows.

For the flows where the free-stream turbulence intensity is low a second self-similar region (II) was found (see [25]). The velocity distribution in this region, adjacent to the free stream is also described by a scaling law

$$
\phi=B \eta^{\beta},
$$


with the parameters different from those of the law (29). The intersection of these two selfsimilar regions is sharp. The Reynolds number for the region (I) was defined as $\operatorname{Re}=U \Lambda / \nu$, where $U$ is the free stream velocity, and $\Lambda$ is the length scale defined by the procedure described above. Analysis showed (see [26]) that for the flows under investigation $\Lambda$ is approximately $1.5 \div 1.6$ times the wall region thickness defined by the sharp intersection of two regions (I) and (II).

The validity of the scaling law (18) for the lower self-similar region of the boundary layer flows constitutes a strong argument in favour of its validity for a wide class of wall-bounded turbulent shear flows at large Reynolds numbers.

The nature of the second self-similar region adjacent to the free stream is not yet clear. For zero-pressure-gradient boundary layer flows with low free stream turbulence the power $\beta$ in the scaling law was found close to 0.2 . The data for non-zero-pressure-gradient flows are substantially less numerous. Recently the data of I. Marušić and A. E. Perry [19] appeared on the Internet in a digital form. Processing of these data (see [27]) confirmed the 'broken line' structure of the velocity distribution in the boundary layer: two self-similar regions with a sharp intersection (see Figure 7). It demonstrated also a substantial variation of the power $\beta$. In addition to the parameter Re governing the structure of the region (I), the power $\beta$ and the coefficient $B$ in the scaling law (32) were found to depend on the dimensionless parameter

$$
P=\nu \partial_{x} p / \rho u_{*}^{3},
$$

where $\partial_{x} p$ is the pressure gradient.

According to the basic assumptions of the models of the turbulent boundary layers proposed previously by F. H. Clauser [20] and D. E. Coles [21], which are widely accepted and used, the transition from the flow in the wall region described by the universal logarithmic law to the external flow is smooth. According to the new model, presented above, if the turbulence in the external flow is small, then the intermediate region between the viscous sublayer and the external flow consists of two Reynolds-number-dependent self-similar regions separated by a sharp boundary. If the external flow is turbulized, the transition from the wall region where the law (18) is valid to the external flow is smooth, but he flow outside the wall region is strongly influenced by the turbulence of the external flow which should be explicitly taken into account in the analysis of this region.

In conclusion I can say the following. The obtained results are significant because engineers have long known that conclusions drawn from the universal logarithmic law, for example, drag coefficient, are unreliable, and they use instead "corrected" empirical functions that fit the data; now the empirical fits can be derived from a better law. However, the main significance of this work lies in its impact on theory: it connects and also solidifies our understanding of key issues of turbulence and has broad implications. 


\section{References}

[1] von Kármán, Th. (1930). Mechanische Ähnlichkeit und Turbulenz. Proc. 3rd Int. Congr. Appl. Mech. Stockholm, C. W. Oseen and W. Weibull (eds.). AB Sveriges Lifografska Tryckenier, vol. 1, 85-93.

[2] Prandtl, L. (1932). Zur turbulenten Strömung in Röhren und längs Platten. Ergebn. Aerodyn. Versuchsanstalt, Göttingen B4, 18-29.

[3] Kolmogorov, A. N. (1941). The local structure of turbulence in incompressible fluids at very high Reynolds numbers. Doklady, USSR Ac. Sci. 30(4), 299-303.

[4] Obukhov, A. M. (1941). On the distribution of energy in the spectrum of a turbulent flow. Doklady, USSR Ac. Sci. 32(1), 22-24.

[5] Kolmogorov, A. N. (1991). Selected works, vol. 1 Mathematics and Mechanics, V. M. Tikhominov (ed.). Kluwer, Dordrecht et al.

[6] Landau, L. D. and Lifshitz, E. M. (1987). Fluid Mechanics, 2nd edition. Pergamon Press, London, New York.

[7] Monin, A. S. and Yaglom, A. M. (1971). Statistical Fluid Mechanics. Mechanics of Turbulence, vol. 1. MIT Press, Cambridge, London.

[8] Zagarola, M. V. (1996). Mean flow scaling in turbulent pipe flow. PhD Thesis, Princeton University.

[9] Barenblatt, G. I. (1996). Scaling, Self-Similarity, and Intermediate Asymptotics. Cambridge University Press.

[10] Schlichting, H. (1968). Boundary Layer Theory, 6th edition. McGraw-Hill, New York.

[11] Nikuradse, J. (1932). Gesetzmässigkeiten der turbulenten Strömung in glatten Röhren. VDI Forschungscheftg No. 356.

[12] Kline, S. J., Reynolds, W. C., Schraub, F. A. and Rundstadtler, P. W. (1967). The structure of turbulent boundary layers. J. Fluid Mech. 30(4), 741-774.

[13] Izakson, A. (1937). Formula for the velocity distribution near a wall. J. Exp. Theor. Physics 7, 919-924.

[14] Millikan, C. B. (1939). A critical discussion of turbulent flows in channels and circular tubes. In: J. P. Den Hartog and H. Peters (eds.), Proc. 5th Int. Congress Appl. Mech., Cambridge, Mass., 386-392. 
[15] von Mises, R. (1941). Some remarks on the laws of turbulent motion in tubes. In: Th. von Kármán, Anniversary Volume. CalTech Press, Pasadena, 317-327.

[16] Erm, L. P. and Joubert, P. N. (1991). Low Reynolds-number turbulent boundary layers. J. Fluid Mech. 230, 1-44.

[17] Krogstad, P.-A. and Antonia, R. A. (1999). Surface roughness effects in turbulent boundary layers. Exp. in Fluids 27, 450-460.

[18] Fernholz, H. H. and Finley, P. J. (1996). The incompressible zero-pressure gradient turbulent boundary layer: an assessment of the data. Progr. Aeros. Sci. 32, 245-311.

[19] Marušić, I. and Perry, A. E. (1995). A wall-wake model for the turbulence structure of boundary layers. Part 2. Further experimental support. J. Fluid Mech. 298, 389-407 (http://www.mame.mu.oz.au/ivan)

[20] Clauser, F. H. (1956). The turbulent boundary layer. Adv. Appl. Mech. 4, Academic Press, New York, 2-52.

[21] Coles, D. E. (1956). The law of the wake in a turbulent boundary layer. J. Fluid Mech. 1(3), 191-226.

[22] Barenblatt, G. I., Chorin, A. J. and Prostokishin, V. M. (1997). Scaling laws in fully developed turbulent pipe flow. Appl. Mech. Rev. 50, 413-429.

[23] Chorin, A. J. (1998). New perspectives in turbulence. Quarterly of Appl. Math. XIV(4), $767-785$.

[24] Barenblatt, G. I. (1999). Scaling laws for turbulent wall-bounded shear flows at very large Reynolds numbers. J. Eng. Math. 36, 361-384.

[25] Barenblatt, G. I., Chorin, A. J. and Prostokishin, V. M. (2000). Self-similar intermediate structures in turbulent boundary layers at large Reynolds numbers. J. Fluid Mech. 410, $263-283$.

[26] Barenblatt, G. I., Chorin, A. J. and Prostokishin, V. M. (2000). Characteristic length scale of the intermediate structure in zero-pressure-gradient boundary layer flow. Proc. US Nat. Acad. Sci. 97, 3799-3802.

[27] Barenblatt, G. I., Chorin, A. J. and Prostokishin, V. M. (2002). A model of turbulent boundary layer with a non-zero pressure gradient. Proc. US Nat. Acad. Sci. 99, 57725776. 


\section{Figure captions}

Figure 1. Flow in a long cylindrical pipe: structure at large Reynolds number: 1) viscous sublayer, 2) near-axis region, and 3) intermediate region.

Figure 2. The Princeton data (Zagarola [8]) obtained in a high-pressure pipe confirm the splitting of the experimental data according to their Reynolds numbers and the rise of the curves above their envelope in the $(\ln \eta, \phi)$-plane. The solid line is the envelope; the curves turn at the center of the pipe. The splitting and form of the curves agree with the scaling law, and are incompatible with the von Kármán-Prandtl universal logarithmic law.

Figure 3. Velocity distribution in smooth pipes can be represented with good accuracy by a power law with Reynolds-number-dependent exponent practically over the whole pipe cross-section. Here $R=d / 2, U$ is the velocity at the center line (Schlichting [10]).

Figure 4. The experimental data of Nikuradze (Nikuradze [11]) in the coordinates $(\ln \eta, \psi)$ at $\eta>30$ lie close to the bisectrix of the first quadrant, confirming the scaling law.
(1) $\Delta, \quad \operatorname{Re}=4 \times 10^{3}$;
(2) $\boldsymbol{\Lambda}, \quad \operatorname{Re}=6.1 \times 10^{3}$;
(3) $\circ, \quad \operatorname{Re}=9.1 \times 10^{3}$;
$(4) \bullet, \quad \operatorname{Re}=1.67 \times 10^{4}$;
(5) $\square, \quad \operatorname{Re}=2.33 \times 10^{4}$;
(6) $\mathbf{\square}, \quad \operatorname{Re}=4.34 \times 10^{4}$;
(7) $\nabla, \quad \operatorname{Re}=1.05 \times 10^{5}$;
(8) $\mathbf{\nabla}, \quad \operatorname{Re}=2.05 \times 10^{5}$;
(9) $\cup, \quad \operatorname{Re}=3.96 \times 10^{5}$;
(10) $\cup, \quad R e=7.25 \times 10^{5}$;
(11) $\diamond, \quad \operatorname{Re}=1.11 \times 10^{6}$;
(12) $\quad \operatorname{Re}=1.536 \times 10^{6}$;
(13),$+ \quad \operatorname{Re}=1.959 \times 10^{6}$;
(14) $\times, \quad \operatorname{Re}=2.356 \times 10^{6}$;
(15) $\cap, \quad \operatorname{Re}=2.79 \times 10^{6}$;
(16) $\cap, \quad \operatorname{Re}=3.24 \times 10^{6}$;

Figure 5. (a) The experiments by Erm and Joubert [16], $\operatorname{Re}_{\theta}=2,788$. Both self-similar intermediate regions (I) and (II) are clearly seen. (b) The experiments of Krogstad and Antonia [17], $\operatorname{Re}_{\theta}=12,570$. Both self-similar intermediate regions (I) and (II) are clearly seen. (c) The experiments of Petrie, Fontaine, Sommer and Brugart obtained by scanning the graphs in Fernholz and Finley [18], $\operatorname{Re}_{\theta}=35,530$. The first self-similar region (I) is revealed; the second self-similar region is not revealed. (d) The experiments of Smith obtained by scanning the graphs in Fernholz and Finley [18], $\operatorname{Re}_{\theta}=12,990$. The first self-similar region (I) is clearly seen, the second region (II) can be revealed.

Figure 6. The experiments by Erm and Joubert [16] $(*)$, Krogstad and Antonia [17] $(\triangle)$, Smith $(\square)$ and Peetrie et al $(\nabla)$ collapse on the bisectrix of the first quadrant in $\ln \eta, \psi$ plane in accordance with the universal form (31) of the scaling law (25). 
Figure 7. The mean velocity profiles in bilogarithmic coordinates in the series of experiments of Marušić [19] for $U=30 \mathrm{~m} / \mathrm{s}$, adverse pressure gradient.
(1) $\operatorname{Re}_{\theta}=19,133, \quad \ln \operatorname{Re}_{\Lambda}=8.83, \quad P=7.04 \cdot 10^{-3}, \quad \beta=0.388$
(2) $\operatorname{Re}_{\theta}=16,584, \quad \ln \operatorname{Re}_{\Lambda}=10.18, \quad P=5.79 \cdot 10^{-3}, \quad \beta=0.346$
(3) $\operatorname{Re}_{\theta}=14,208, \quad \ln \operatorname{Re}_{\Lambda}=10.20, \quad P=4.2 \cdot 10^{-3}, \quad \beta=0.306$
(4) $\operatorname{Re}_{\theta}=10,997, \quad \ln \operatorname{Re}_{\Lambda}=10.31, \quad P=2.86 \cdot 10^{-3}, \quad \beta=0.247$
(5) $\operatorname{Re}_{\theta}=8,588, \quad \ln \operatorname{Re}_{\Lambda}=10.323, \quad P=1.75 \cdot 10^{-3}, \quad \beta=0.207$
(6) $\operatorname{Re}_{\theta}=6,430, \quad \ln \operatorname{Re}_{\Lambda}=10.51, \quad P=0, \quad \beta=0.190$

The "broken-line" structure of the profiles is clearly seen and regions I and II are clearly distinguishable. 


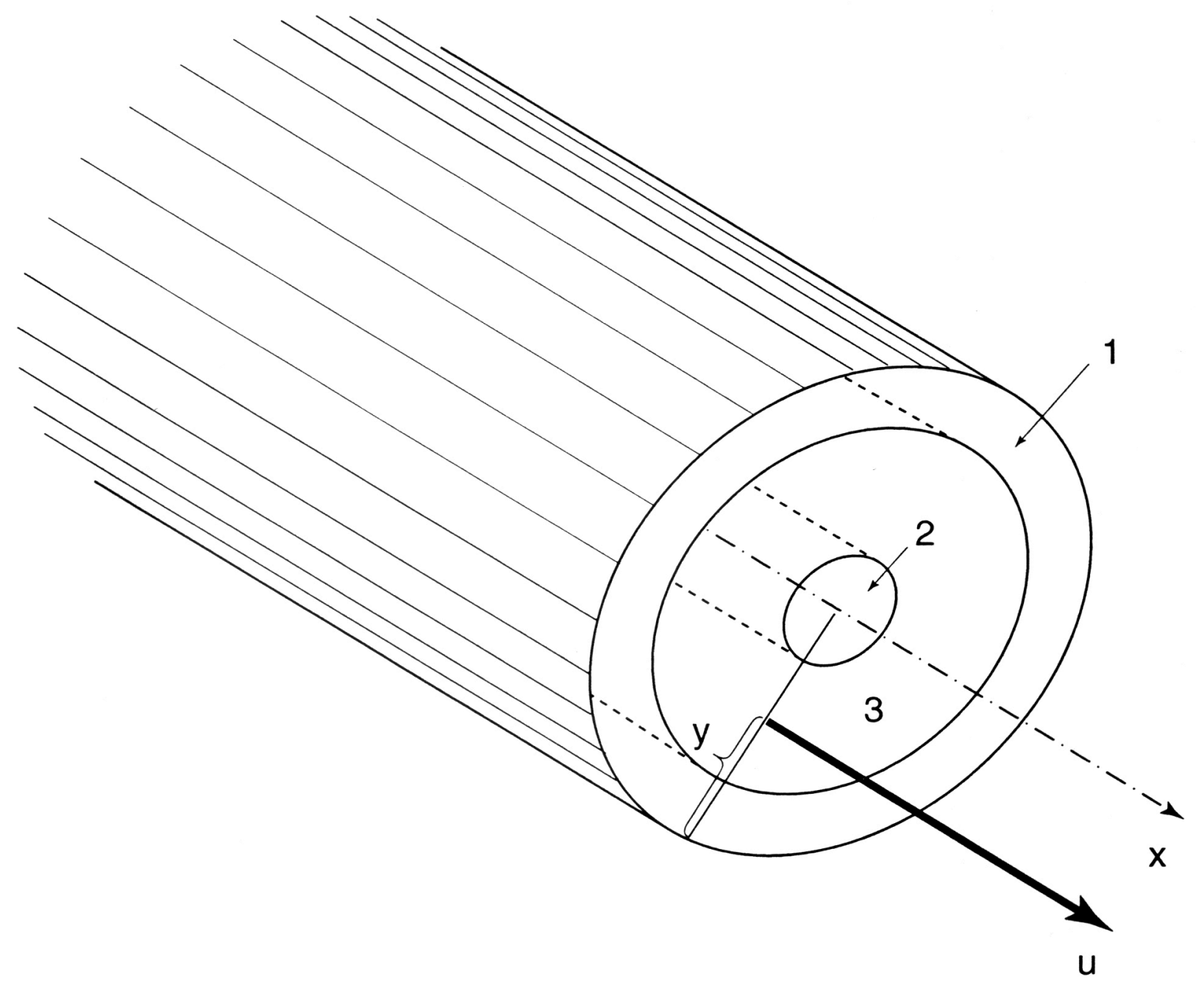

XBD 9611-05578.ILR

Figure 1 


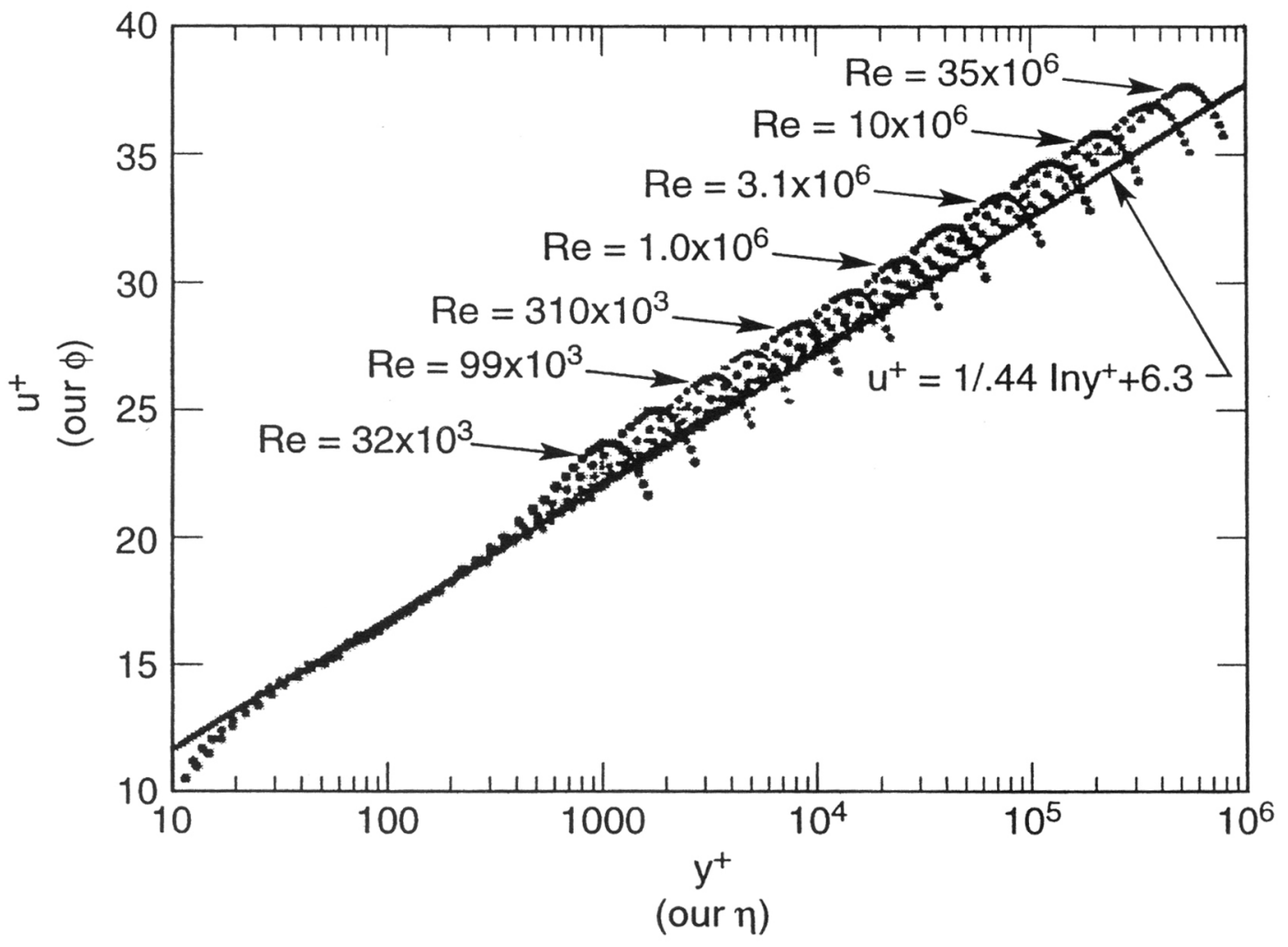

Figure 2 

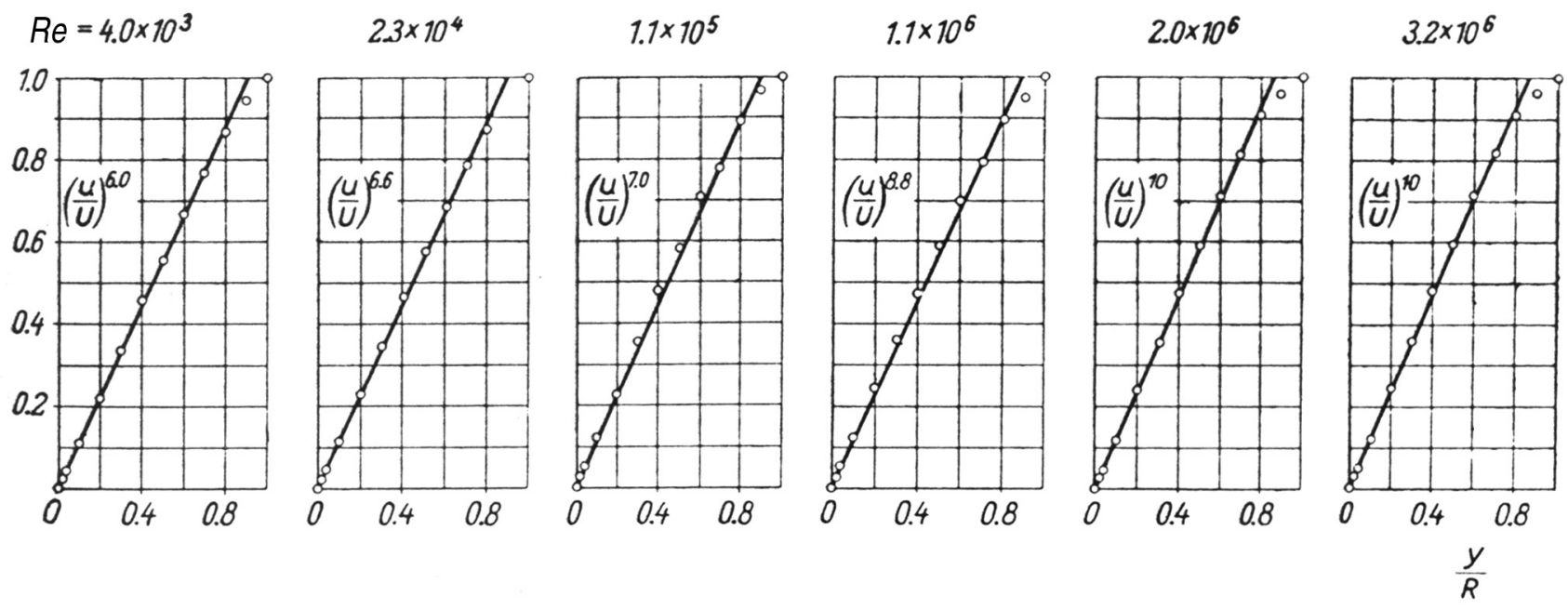

Figure 3 


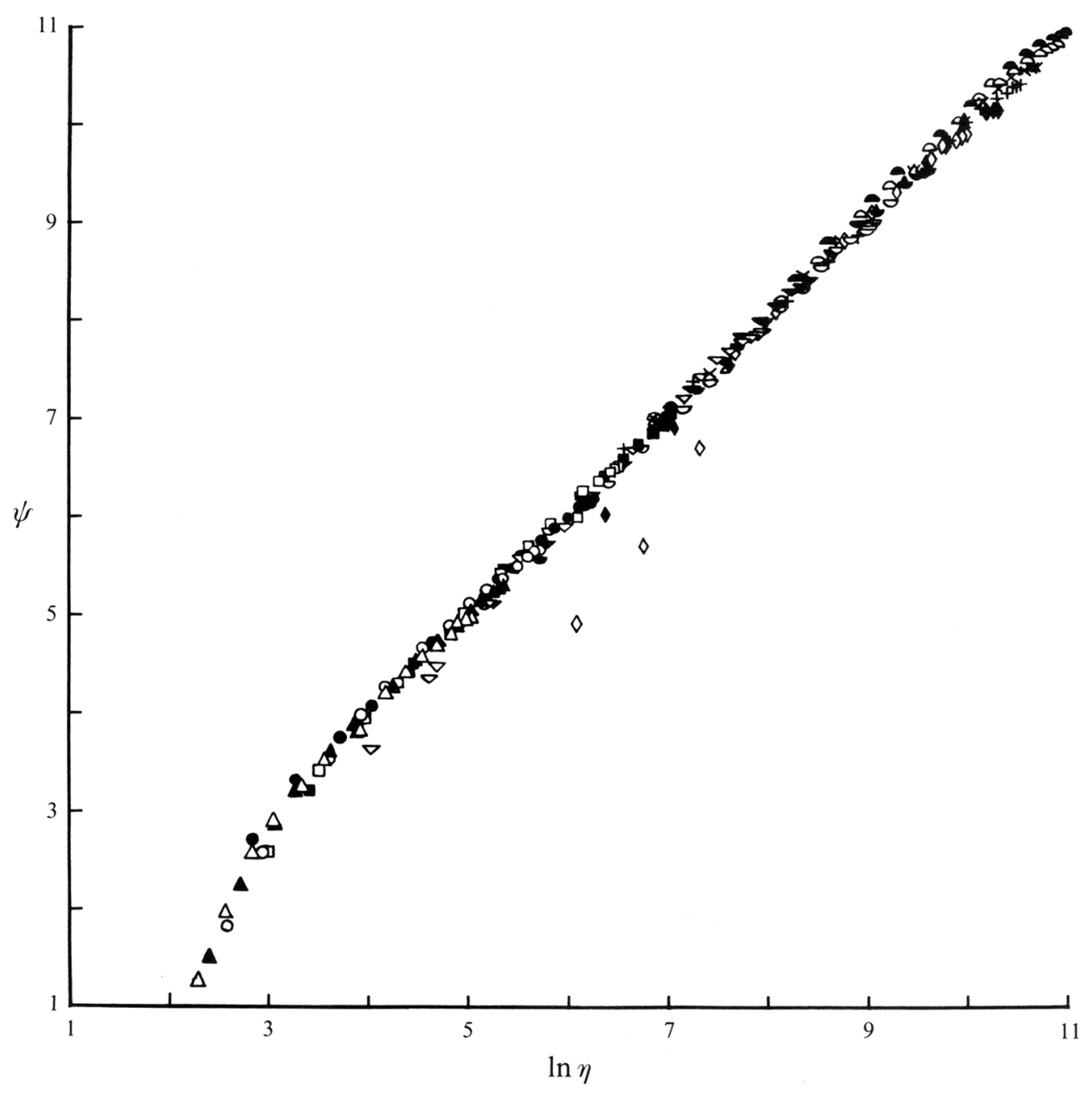

Figure 4 

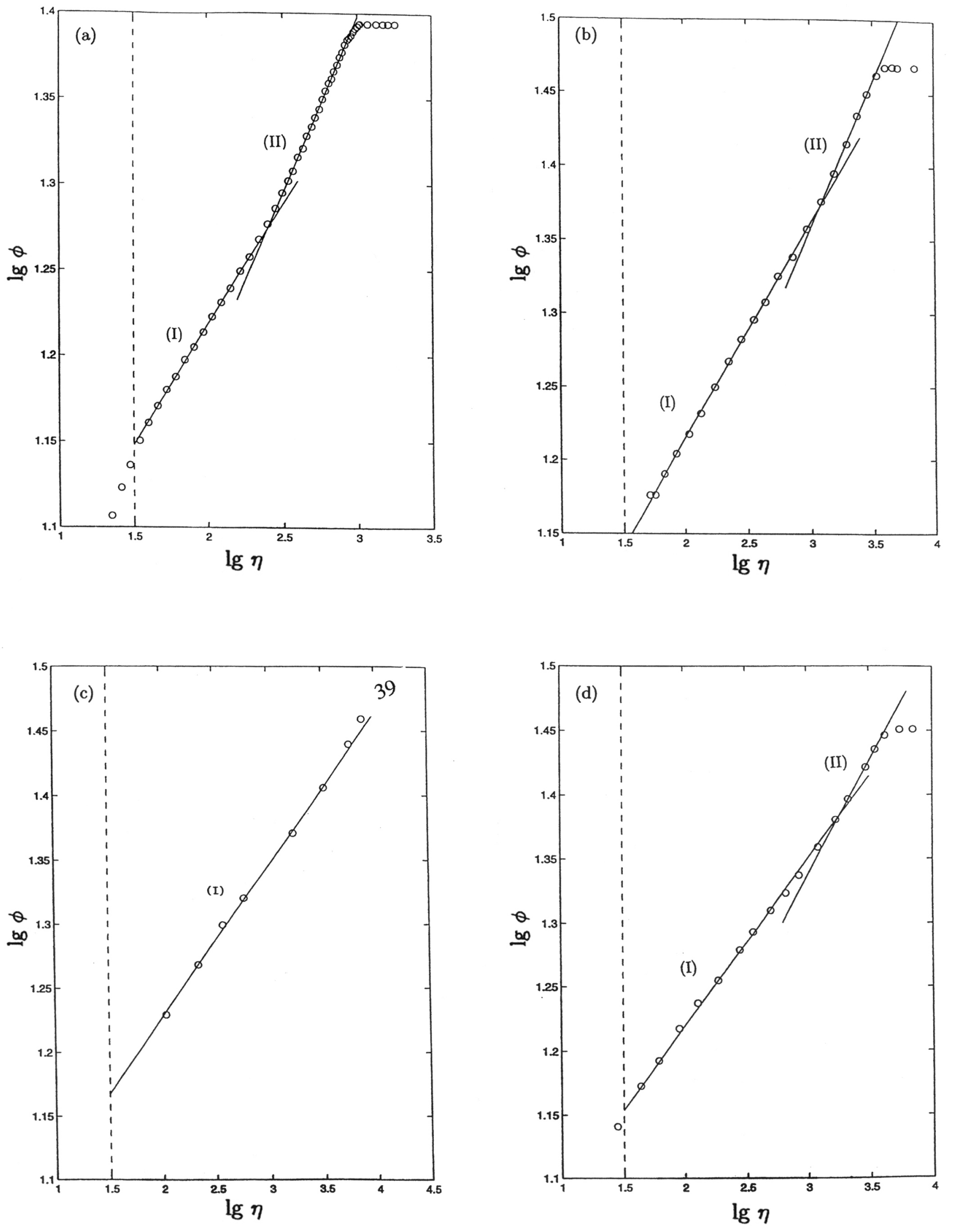

Figure 5 


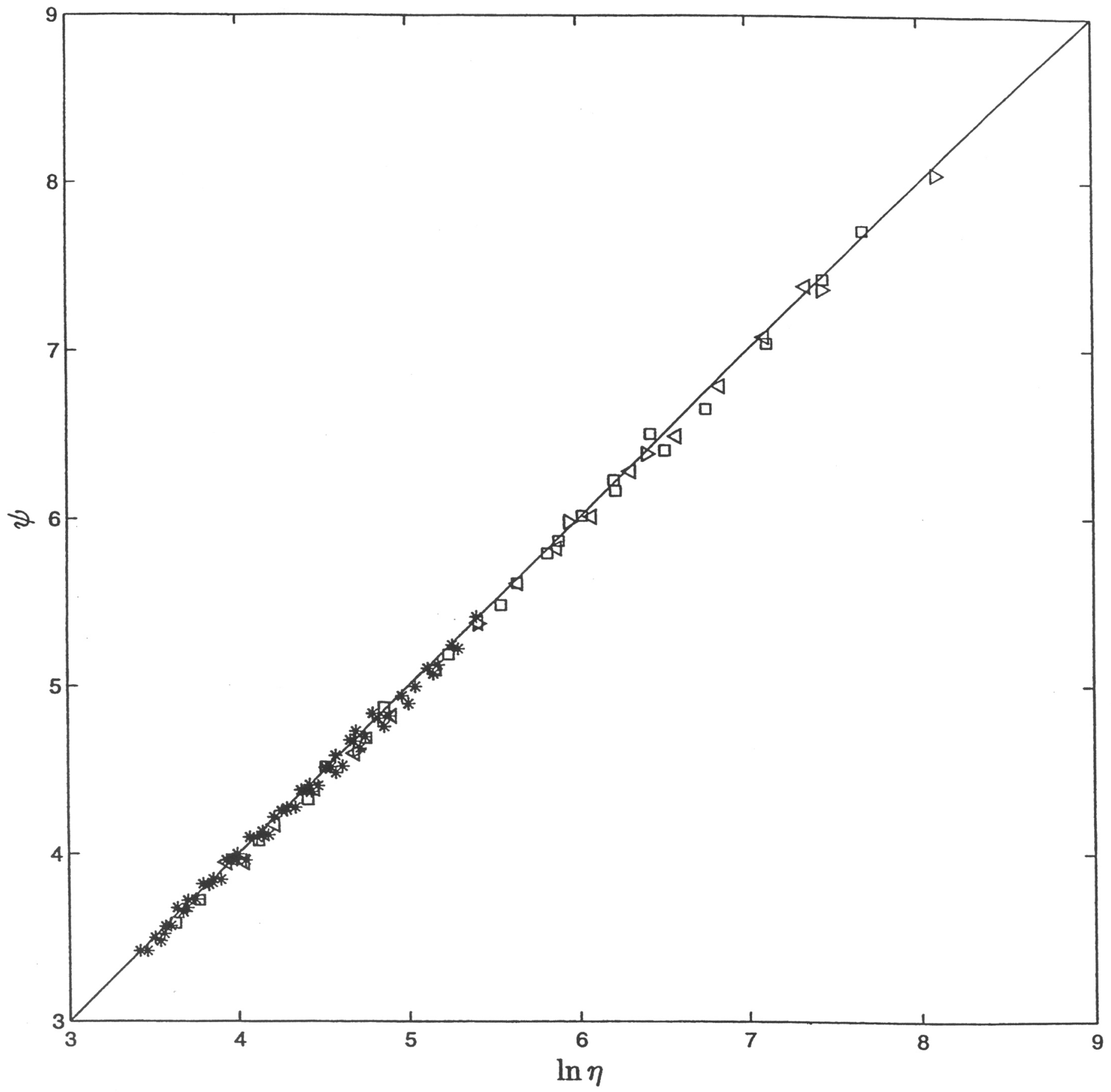

Figure 6 


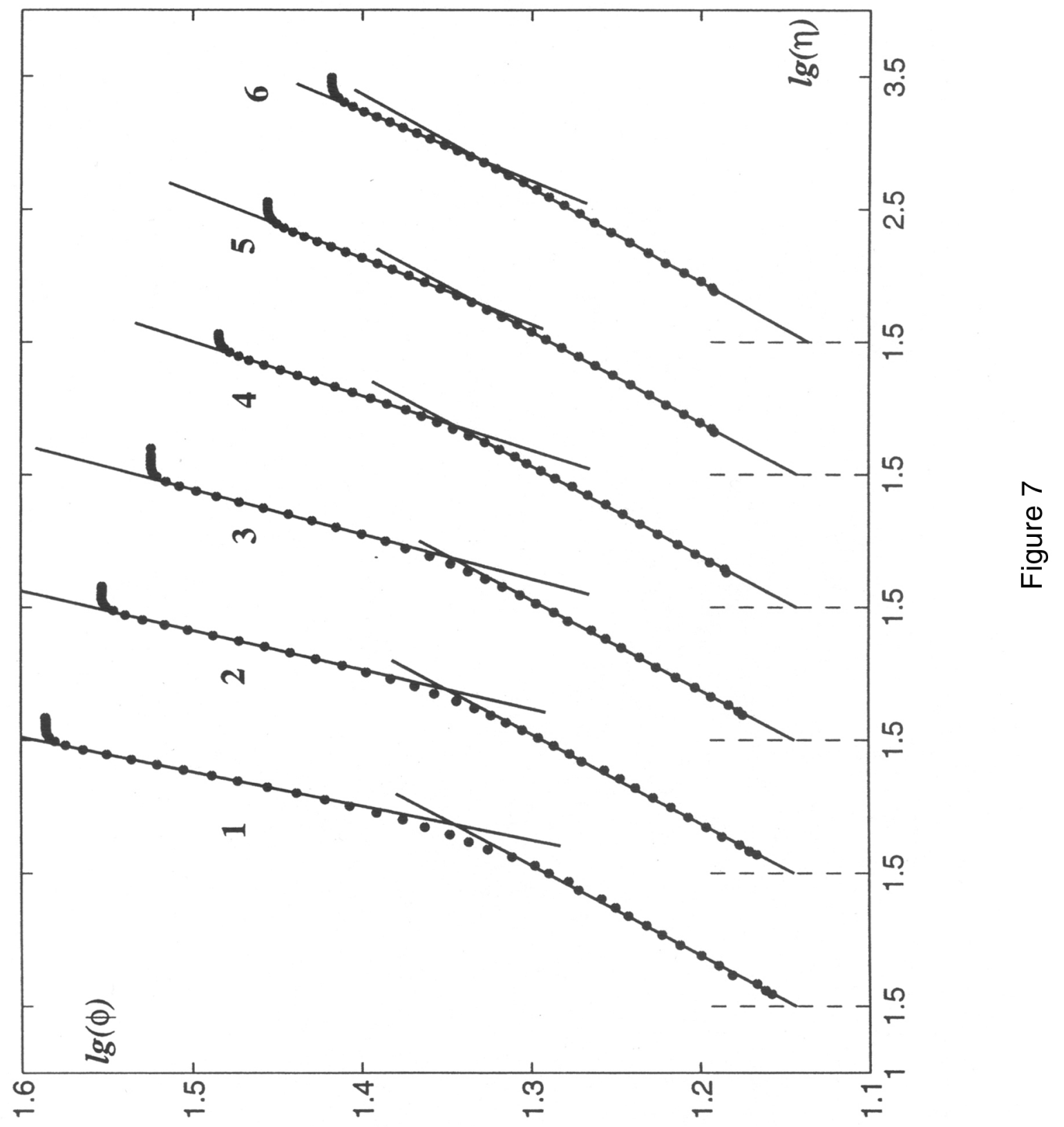

